HIFAN 1673

\title{
SONOLUMINESCENCE TEST FOR EQUATION OF STATE IN WARM DENSE MATTER
}

\author{
Siu-Fai Ng1, 2, J. J. Barnard3, P. T. Leung, S. S. Yu1, 2 \\ 1. Physics Department, The Chinese University of Hong Kong, Hong Kong SAR, China \\ 2. Lawrence Berkeley National Laboratory, California, USA \\ 3. Lawrence Livermore National Laboratory, California, USA
}

Accelerator Fusion Research Division

Ernest Orlando Lawrence Berkeley National Laboratory

University of California

Berkeley, California 94720

August 2008

This work was supported by the Director, Office of Science, Office of Fusion Energy Sciences, of the U.S. Department of Energy under Contract No. DE-AC02-05CH11231. 


\title{
SONOLUMINESCENCE TEST FOR EQUATION OF STATE IN WARM DENSE MATTER
}

\author{
Siu-Fai Ng ${ }^{1,2}$, J. J. Barnard ${ }^{3}$, P. T. Leung ${ }^{1}$, S. S. Yu ${ }^{1,2}$ \\ 1. Physics Department, The Chinese University of Hong Kong, Hong Kong SAR, China \\ 2. Lawrence Berkeley National Laboratory, California, USA \\ 3. Lawrence Livermore National Laboratory, California, USA
}

\begin{abstract}
In experiments of Single-bubble Sonoluminescence (SBSL), the bubble is heated to temperatures of a few $\mathrm{eV}$ in the collapse phase of the oscillation. Our hydrodynamic simulations show that the density inside the bubble can go up to the order of $1 \mathrm{~g} / \mathrm{cm}^{3}$, and the electron density due to ionization is $10^{21} / \mathrm{cm}^{3}$. So the plasma coupling constant is found to be around 1 and the gas inside the bubble is in the Warm Dense Matter (WDM) regime. We simulate the light emission of SL with an optical model for thermal radiation which takes the finite opacity of the bubble into consideration. The numerical results obtained are compared to the experimental data and found to be very sensitive to the equation of state used. As theories for the equation of state, as well as the opacity data, in the WDM regime are still very uncertain, we propose that SL may be a good low-cost experimental check for the EOS and the opacity data for matter in the WDM regime.
\end{abstract}

\section{Introduction}

Single-bubble Sonoluminescence (SBSL or SL), which was discovered in 1989, is a phenomenon of periodic light emission by an oscillating gas bubble trapped in the pressure anti-node of a standing ultrasound wave in water (or other fluids). Several experiments have shown that the width of the emitted light pulse is of the order of 100 ps with peak power of the order of $10 \mathrm{~mW} .^{1-3}$

Our simulations, as well as the results in many papers ${ }^{1-7}$, show that the temperature inside the bubble can go up to a few $\mathrm{eV}$ and the density is around $1 \mathrm{~g} / \mathrm{cm}^{3}$ in the collapse phase. At this temperature and density, it is believed that the bubble has entered into the Warm Dense Matter (WDM) regime, a regime of significant theoretical interest because of the strongly coupled effect between ions and atom. (In general, WDM is defined to be the state of material at temperature of the order of 0.1 to $20 \mathrm{eV}$ and density of 0.1 to 10 solid density, with plasma coupling constant of around 1.) Since the equation of state, opacity and other properties in the WDM regime are still very uncertain, many experiments (such as high intensity heavy ion beam target experiments) have been proposed to probe these properties empirically.

Since the light pulse emitted in the collapse phase of SL can be measured quite precisely, we propose that SL may be a good and easy check for the EOS and opacity in WDM.

\section{Sonoluminescence Simulations}

In our SL simulations, we used the 1-D code $\mathrm{DISH}^{8}$ to model hydrodynamics inside the bubble. The DISH code, originally written for the slab geometry, was modified for the (assumed) perfect spherical symmetry of SL.

For the outer boundary condition for the bubble, we approximate the dynamics of the water outside the bubble by the commonly used Rayleigh-Plesset (RP) equation ${ }^{9}$ :

$$
\begin{array}{r}
\rho\left[\left(1-\frac{2 \dot{R}}{c}\right) R \ddot{R}+\frac{3}{2}\left(1-\frac{4 \dot{R}}{3 c}\right) \dot{R}^{2}\right]=P_{\text {gas }}-P_{\text {apply }} \\
-P_{0}+\frac{R}{c}\left(1-\frac{\dot{R}}{c}\right) \frac{d}{d t} P_{g a s}-4 \eta \frac{\dot{R}}{R}-2 \frac{\sigma}{R}
\end{array}
$$

where $\rho, c, \eta$ and $\sigma$ are density, speed of sound, viscosity and surface tension of the water, $R$ is the radius of the bubble as a function of time, $P_{0}$ is the constant ambient pressure $\left(P_{0}=1 \mathrm{~atm}\right), P_{a p p l y}$ is the applied pressure ( $\left.P_{a p p l y}=-P_{a} \sin \omega t\right)$, and $P_{\text {gas }}$ is the gas pressure just inside the bubble wall, which depends on the hydrodynamic evolution of the bubble and the equation of state used.

Our simulation includes thermal conduction with Argon thermal conductivity $\kappa=0.009+0.00032 T \quad{ }^{10}$, 
where $\kappa$ is in $\mathrm{W} / \mathrm{m} \mathrm{K}$ and $T$ is in $\mathrm{K}$.

To calculate the radiation emitted (power emitted per wavelength interval), we use the integral form of the radiation transport equation ${ }^{11}$ :

$$
\begin{aligned}
P_{\lambda}(t)= & \int_{0}^{R} 16 \pi^{2} \kappa_{\lambda}^{t o t}(r, t) I_{\lambda}^{P l}(r, t) \\
& \times \exp \left(-\int_{r}^{R} \kappa_{\lambda}^{t o t}\left(r^{\prime}\right) d r^{\prime}\right) r^{2} d r
\end{aligned}
$$

where $\kappa_{\lambda}^{t o t}$ is the total absorption coefficient and $I_{\lambda}^{P l}$ is the spectral emission by Planck blackbody radiation:

$$
I_{\lambda}^{P l}=\frac{2 h c^{2}}{\lambda^{5}[\exp (h c / \lambda k T)-1]}
$$

We consider the free-free interactions between electrons and ions and between electrons and atoms as the two dominant processes for radiation absorption ${ }^{5,11,12}$. So $\kappa_{\lambda}^{t o t}=\kappa_{\lambda}^{\mathrm{ff}+}+\kappa_{\lambda}^{\mathrm{ff} 0}$ and:

$$
\begin{aligned}
& \kappa_{\lambda}^{\mathrm{ff}+}=\frac{4}{3}\left(\frac{2 \pi}{3 m_{e} k T}\right)^{1 / 2} \frac{Z^{2} e^{6} n_{i} n_{e} \lambda^{3}}{\left(4 \pi \varepsilon_{0}\right)^{3} h c^{4} m_{e}} \\
& \kappa_{\lambda}^{\mathrm{ff} 0}=\frac{4 e^{2} n_{e} n_{0} \lambda^{2}}{\left(4 \pi \varepsilon_{0}\right)^{3} \pi^{3 / 2} c^{3}}\left(\frac{2 k T}{m_{e}}\right)^{3 / 2}\left(c+\frac{d}{3 k T}\right)
\end{aligned}
$$

where $n_{0}, n_{i}$ and $n_{e}$ are the density of atoms, ions and electrons respectively, and are dependent on the EOS assumed. $Z$ is the charge of the ions. $c$ and $d$ are constants equal to $1.6 \times 10^{-20} \mathrm{~m}^{2} / \mathrm{eV}$ and $-0.6 \times 10^{-20} \mathrm{~m}^{2}$.

\section{Equation of State}

Results of sonoluminescence simulations depend on the equation of state (EOS) used but the EOS in WDM regime is still uncertain. Here we use two different equations of state that are commonly used in WDM simulations: QEOS and Saha-based EOS.

\section{A. QEOS}

QEOS (The quotidian equation of state) ${ }^{13}$ is a well-known equation of state model for hydrodynamic simulations of
WDM and other high-pressure phenomena. In QEOS, the electronic properties are obtained from a modified Thomas-Fermi statistical model, while the ion thermal motion is described by a multiphase equation of state combining Debye, Gruneisen, Lindemann, and fluid-scaling laws. QEOS can give smooth predictions for ionization state, pressure, energy, entropy and Helmholtz free energy for use in hydrodynamic simulations.

\section{B. Saha-based EOS}

We also use the well-known Saha equation to get the ionization of the gas $^{14,15}$ :

$$
\frac{n_{e} n_{\zeta}}{n_{\zeta-1}}=\frac{2 Q_{\zeta}}{Q_{\zeta-1}}\left(\frac{2 \pi m_{e} k T}{h^{2}}\right)^{3 / 2} \exp \left(-\frac{E_{\zeta}^{i o n}-\Delta E}{k T}\right)
$$

This equation gives the ratio of the ion density of charge state $\zeta$ and $\zeta-1$ in terms of electron density $n_{e}$, temperature $T$ and ionization energy $E_{\zeta}^{i o n} . \Delta E$ is the continuum lowering term to reduce the energy needed for ionization due to the interaction within ions and between ions and electrons. $Q_{\zeta}$ is the partition function of the individual ion with charge $+\zeta$. Mathematically, the infinite series $Q_{\zeta}$ diverges for an isolated ion. To converge the series, we truncate the series when the excitation energy $E_{\zeta m}^{e x} \geq E_{\zeta+1}^{i o n}-\Delta E$

We consider the ion sphere model to calculate $\Delta E^{14}$. In an ion sphere with an infinitely small $+\zeta$ ion at origin and $\zeta$ free electrons distributed uniformly in the sphere, the net charge in the sphere is zero. Assume that the charges outside the sphere neutralize each other and have no effect on the potential at the origin, we can calculate the extra potential energy on that ion due to the free electrons in the sphere:

$$
\Delta E=\frac{3}{2}\left(\frac{4 \pi}{3}\right)^{1 / 3} \zeta e^{2} n_{i}^{1 / 3}
$$


We also include the hard core effect due to interactions between gas particles in the Saha-based EOS model ${ }^{16}$. Finally, we obtain the pressure and energy per unit mass (EOS):

$$
\begin{aligned}
P=( & \left.n+n_{e}\right) k T-\frac{1}{3} n_{e} \Delta E-(a-b k T) n^{2} \\
e=\frac{1}{\rho} & {\left[\frac{3}{2}\left(n+n_{e}\right) k T-a n^{2}+\sum_{\zeta=0} n_{\zeta}\left\langle E_{\zeta}^{e x}\right\rangle\right.} \\
& \left.+\sum_{\zeta=1} n_{\zeta}\left(\sum_{m=1}^{\zeta} E_{m}^{i o n}-\zeta \Delta E\right)\right]
\end{aligned}
$$

where $n$ is the total density of atoms and ions, $\left\langle E_{\zeta}^{e x}\right\rangle$ is the average excitation energy for the $+\zeta$ ions, constants $b=2 \pi R_{0}^{3} / 3, a=b u_{0}$ where $R_{0}=3.82 \times 10^{-10} \mathrm{~m}$ and $u_{0} / k=120 \mathrm{~K}$ (for Argon).

\section{Simulation Results}

We tried to reproduce the experimental results in a paper by Pecha et. $a l .{ }^{17}$ for an dissolved air in water with driving frequency $f=20 \mathrm{kHz}$, ambient temperature
$T_{0}=8{ }^{\circ} \mathrm{C}$ and driving pressure $P_{a}=1.3$ bar. As stable SL bubble is believed to be a noble gas bubble ${ }^{1,18}$, the SL bubble formed in this case is basically an Argon bubble (air is $1 \%$ Argon). We find by diffusive theories ${ }^{19}$ (using gas concentration to be the Argon concentration) that the ambient radius $r_{0}=4 \mu \mathrm{m}$. Simulations using the two EOS described above were then performed.

Simulations using Saha-based EOS show that at the instant of minimum radius, the temperature at the center will reach its maximum of nearly $25000 \mathrm{~K}$ (Fig. 1A). At this high temperature, the Argon gas will ionize. Fig. 1B shows that the maximum ionization is about $22 \%$. Fig. 2 shows the density, temperature, pressure and charge state profile at the stagnation point. It is obvious that the bubble is not uniform but has a cold and dense shell and a hot core.

When using QEOS to run the simulations, we find that the charge state obtained by QEOS is always much higher than using Saha equation. At room conditions, QEOS will give $25 \%$ ionization, while Saha equation gives 0 until the temperature reaches $5000 \mathrm{~K}$. The maximum central charge state obtained by QEOS is 1.7 (Fig. 3), much higher than

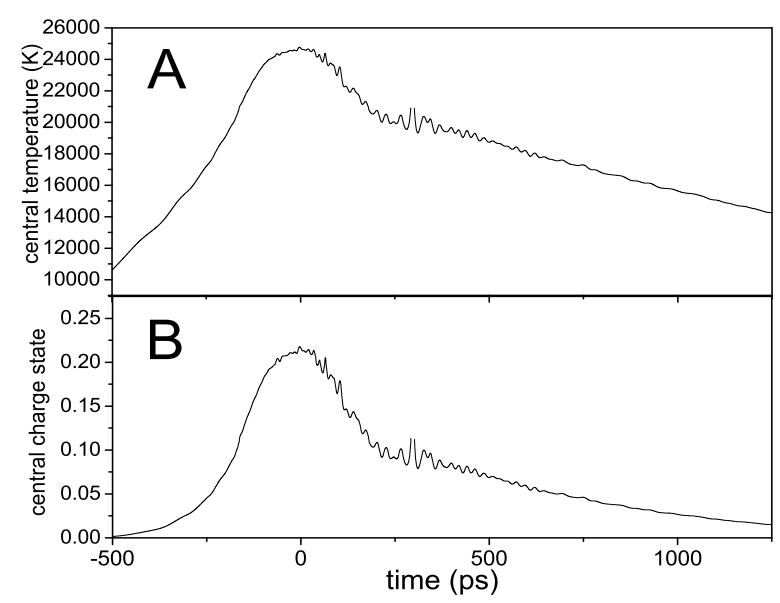

Fig. 1. Results using Saha-based EOS, with time relative to the instant of minimum radius: (A) temperature at the bubble center: maximum is about $25000 \mathrm{~K}$, at $\mathrm{t}=0 \mathrm{ps}$. (B) charge state at bubble center: maximum ionization is about $22 \%$.
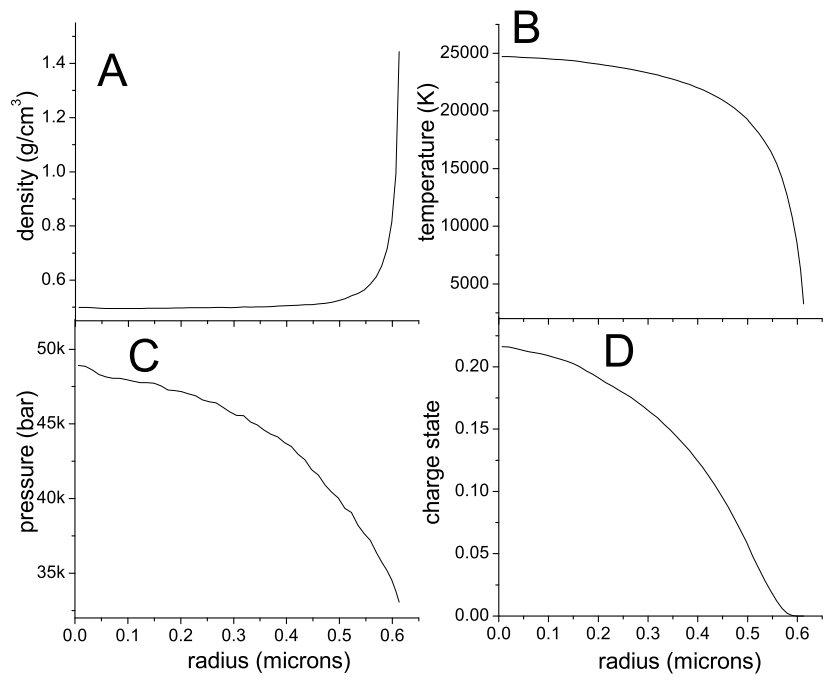

Fig. 2. (A) density, (B) temperature, (C) pressure and (D) charge state profile at minimum radius using Saha-based EOS. 
that obtained by Saha-based EOS (only 0.22). Fig. 4 also shows that the charge state profile is very much different from the Saha-based EOS results, though the density, temperature and pressure are qualitatively the same. For QEOS, there is a highly charged shell but Saha-based EOS gives a zero charge shell.

As the temperature of the bubble at minimum radius is high, the bubble will emit radiation pulse at that time. Using the radiation transport model and blackbody radiation, we calculate the power radiated for the Saha-based EOS and QEOS. Fig. 5A2 shows that the simulation result using the Saha-based EOS matches with the experiment very well. The FWHM calculated (196 ps) is within the error bar of the experimental result (208 ps, $\pm 21 \mathrm{ps})$.

At the same time, QEOS will give a result very different from either the Saha-based EOS or the experiment (Fig. 5B2). At the instant of minimum radius, the power radiated is much less than at other times. So there is no light pulse emitted, clearly disagreeing with the observations.

In Fig. 5A and 5B, we find that QEOS will give much higher absorption coefficients than Saha-based EOS, due to the high charge state, and create an opaque shell, while Saha-based EOS gives a transparent shell. When the bubble collapses, the opaque shell becomes stronger and stronger and absorbs more radiation emitted by the hot core. As a result, when the bubble collapses, less and less radiation can be emitted out of the bubble. This makes the results very unphysical.

\section{Conclusion}

In this paper, we work out a hydrodynamic approach and radiation transport model for SL. We develop a Saha-based EOS with continuum lowering. The electrical interaction between ions and free electrons is modeled, and the hard core potential is included.. Simulations show that this EOS matches with the experiments quite well for the SL problem.

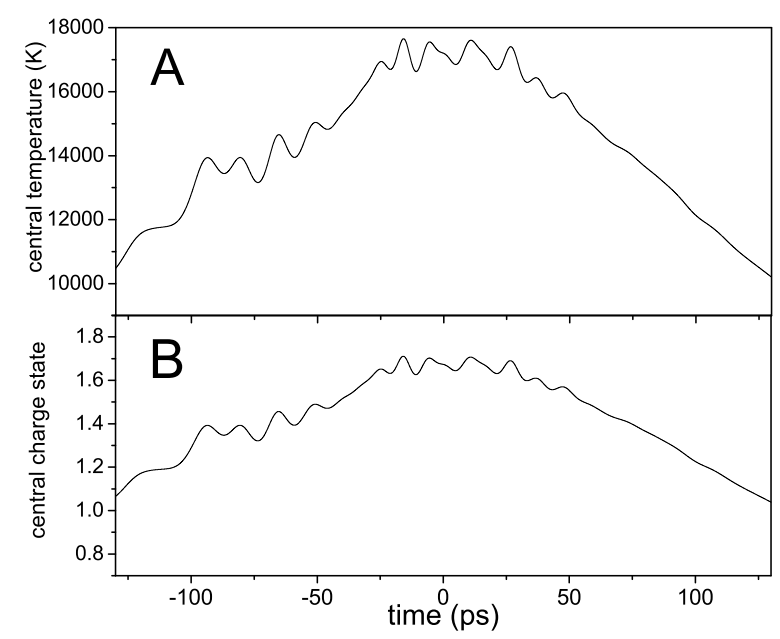

Fig. 3. Results using QEOS: (A) temperature at the bubble center: maximum is about $18000 \mathrm{~K}$. (B) charge state at bubble center can reach about 1.7 around stagnation point, much higher than that for Saha-based EOS at all time.

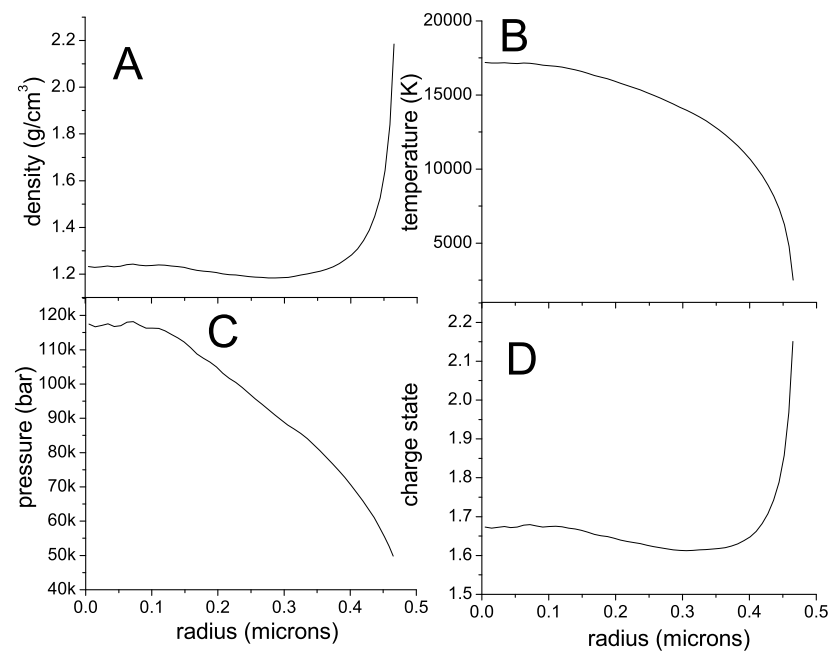

Fig. 4. (A) density, (B) temperature, (C) pressure and (D) charge state profile at minimum radius using QEOS. 
A. Saha-based EOS

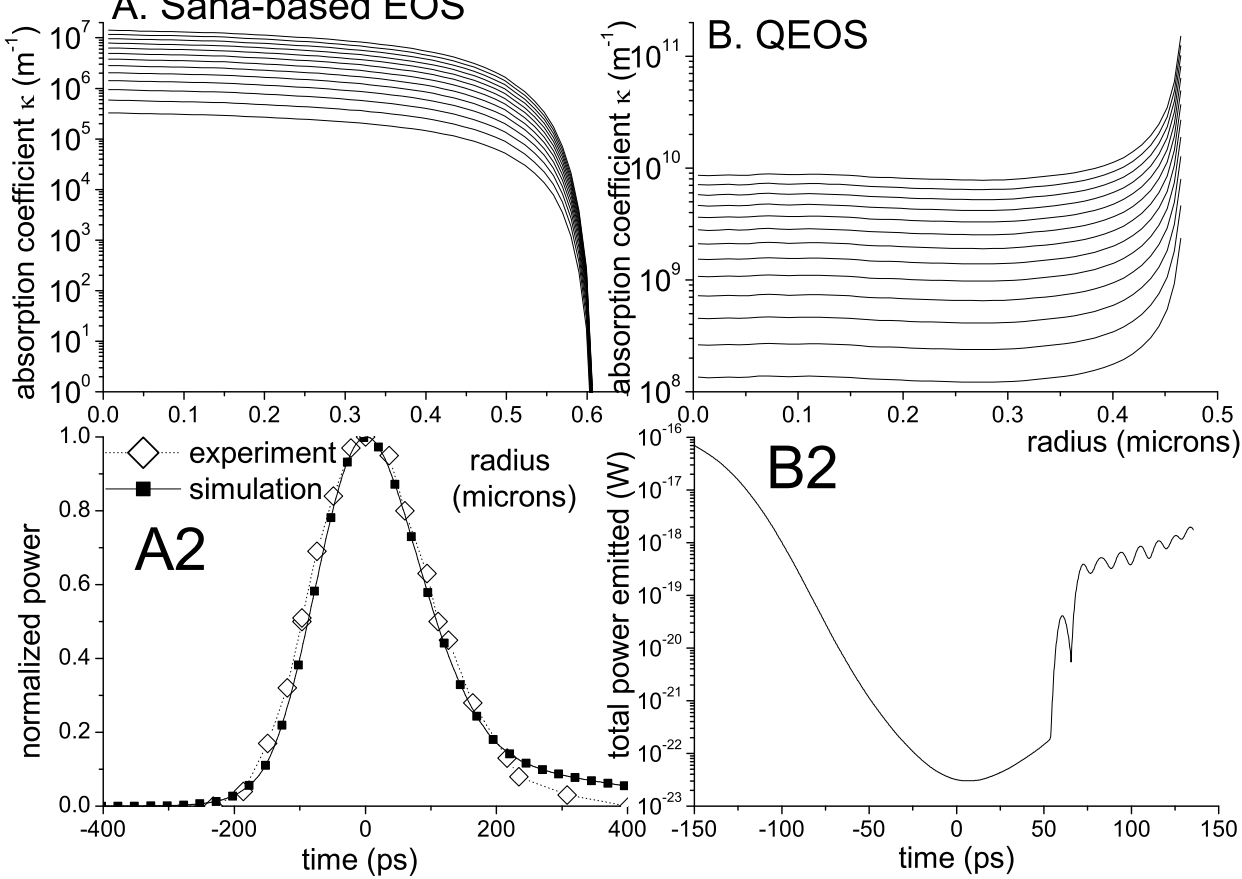

Fig. 5. The absorption coefficients profile (up) and (normalized) power radiated (down) for (A) Saha-based EOS and (B) QEOS.

We also try to use QEOS, a well-known EOS for WDM, for the simulations. But the results are quite unphysical. We believe that though QEOS is widely-used, especially for solid metal, it may not work so well for high ionization energy material, such as Argon gas.

As SL simulation is sensitive to the EOS used, and the EOS for SL (within the WDM regime) is still uncertain, sonoluminescence may offer an easy, low-cost experimental check for the Equation of State in Warm Dense Matter.

\section{Acknowledgments}

We thank M. C. Chu and R. M. More for discussions. We also thank R. M. More for providing DISH code for hydrodynamic simulations, A. J. Kemp and J. Meyer-ter-Vehn for providing MPQeos code for calculating QEOS data, and NIST for providing the energy levels data in the NIST Atomic Spectra Database (website: http://physics.nist.gov/PhysRefData/ASD/index.html).

${ }^{1}$ B. P. Barber, R. A. Hiller, R. Lofstedt, S. J. Puttermsn and K. R. Weninger, Phys. Rep. 281, 65 (1997)
${ }^{2}$ D. Hammer and L. Frommhold, J. Mod. Opt. 48, 239 (2001)

${ }^{3}$ M. P. Brenner, S. Hilgenfeldt and D. Lohse, Rev. Mod. Phys. 74, 425 (2002)

${ }^{4}$ M. C. Chu and D. Leung, J. Phys: Condens. Matter 9, 3387 (1997)

${ }^{5}$ S. Hilgenfeldt, S. Grossmann and D. Lohse, Phys. Fluids 11, 1318 (1999)

${ }^{6}$ S. Hilgenfeldt, S. Grossmann and D. Lohse, Nature (London) 398, 402 (1999)

${ }^{7}$ W. K. Tse and P. T. Leung, Phys Rev. E 73, 056302 (206)

${ }^{8}$ R. M. More, DISH User Manual (2007)

9 F. R. Young, Cavitation (McGraw-Hill, London; New York, 1989)

${ }^{10}$ K. Yasui, Phys. Rev. E 56, 6750 (1997)

${ }^{11}$ C. Y. Ho, L. Yuan, M. C. Chu, P. T. Leung and W. Wei, Phys. Rev. E 65, 041201 (2002)

${ }^{12}$ Y. B. Zel'dovich and Y. P. Raizer, Physics of shock waves and high-temperature hydrodynamic phenomena, Vols. II (Academic Press, New York, 1966) 
13 R. M. More, K. H. Warren, D. A. Young and G. B. Zimmerman, Phys. Fluids 31, 3059 (1988)

14 D. Salzmann, Atomic Physics in Hot Plasmas (Oxford University Press, New York, 1998)

${ }^{15} \mathrm{~S}$. Atzeni and J. Meyer-ter-Vehn, The physics of inertial fusion : beam plasma interaction, hydrodynamics, hot dense matter (Clarendon Press, Oxford, 2004)

16 S. Eliezer, A. Ghatak, H. Hora, An Introduction to Equations of State: Theory and Applications (Cambridge University Press, Cambridge, 1986)

17 R. Pecha, B. Gompf, G. Nick, Z. Q. Wang and W. Eisenmenger, Phys. Rev. Lett. 81, 717 (1998)

${ }^{18}$ R. Hiller, K. Weninger, S. J. Putterman and B. P. Barber, Science 266, 248 (1994)

${ }^{19}$ S. Hilgenfeldt, D. Lohse and M. P. Brenner, Phys. Fluids 8, 2808 (1996) 